\title{
Anomalous Phonon Lifetime Shortening in Paramagnetic CrN Caused by Spin-Lattice Coupling: A Combined Spin and Ab Initio Molecular Dynamics Study
}

\author{
Irina Stockem, ${ }^{1,2, *}$ Anders Bergman, ${ }^{3,4}$ Albert Glensk, ${ }^{2}$ Tilmann Hickel, ${ }^{2}$ Fritz Körmann, ${ }^{2,5}$ Blazej Grabowski, ${ }^{2}$ \\ Jörg Neugebauer, ${ }^{2}$ and Björn Alling ${ }^{1,2}$ \\ ${ }^{1}$ Department of Physics, Chemistry, and Biology (IFM), Linköping University, 58183 Linköping, Sweden \\ ${ }^{2}$ Max-Planck-Institut für Eisenforschung GmbH, 40237 Düsseldorf, Germany \\ ${ }^{3}$ Maison de la Simulation, USR 3441, CEA-CNRS-INRIA-Université Paris-Sud-Université de Versailles, 91191 Gif-sur-Yvette, France \\ ${ }^{4}$ L_Sim, INAC-MEM, CEA, 38000 Grenoble, France \\ ${ }^{5}$ Department of Materials Science and Engineering, Delft University of Technology, Mekelweg 2, 2628 CD Delft, The Netherlands
}

(Received 25 January 2018; published 21 September 2018)

\begin{abstract}
We study the mutual coupling of spin fluctuations and lattice vibrations in paramagnetic $\mathrm{CrN}$ by combining atomistic spin dynamics and ab initio molecular dynamics. The two degrees of freedom are dynamically coupled, leading to nonadiabatic effects. Those effects suppress the phonon lifetimes at low temperature compared to an adiabatic approach. The dynamic coupling identified here provides an explanation for the experimentally observed unexpected temperature dependence of the thermal conductivity of magnetic semiconductors above the magnetic ordering temperature.
\end{abstract}

DOI: 10.1103/PhysRevLett.121.125902

At elevated temperatures, magnetic materials are subject to both spin and lattice excitations. Magnon and phonon spectra, as well as magnetic order-disorder and melting transitions, are often analyzed separately, even though in principle they are distinctly coupled [1]. As an example, the phonon dominated thermal conductivity of magnetic semiconductors $\mathrm{YMnO}_{3}, \mathrm{LuMnO}_{3}$, and $\mathrm{ScMnO}_{3}$ [2], as well as $\mathrm{CrN}$ [3-5], show dramatically different temperature dependences below and above the magnetic transition temperature. Particularly, above the magnetic ordering temperature, the thermal conductivity differs from the temperature dependence of nonmagnetic semiconductors like $\mathrm{MgO}$ [6]. While the phonon based thermal conductivity of nonmagnetic materials typically declines by $1 / T$ at high temperatures due to increasing phonon-phonon interactions [turquoise line in Fig. 1(a)], the thermal conductivity of magnetic semiconductors such as $\mathrm{CrN}$ remains nearly constant above the magnetic transition [red line in Fig. 1(a)]. So far, a well-supported explanation for this behavior has been lacking. We show that in the high temperature paramagnetic phase, nonadiabatic coupling between phonons and spins strongly affects the phonon lifetimes [Fig. 1(b)]. In the high temperature limit, which we consider in this Letter, the single mode approximation of the Boltzmann transport equation $[7,8]$ can be applied. The temperature dependence of the phonon relaxation time can be approximated by the phonon lifetime [7], and it can be directly correlated to the temperature dependence of thermal conductivity. Our findings give an atomistic explanation, and they support the suggestion in the experimental work of Sharma et al. [2], who proposed a dynamical spin-phonon scattering mechanism of acoustic phonons by short-ranged spin fluctuations.

Above the critical Curie or Néel temperature, the paramagnetic state is characterized by a lack of both global

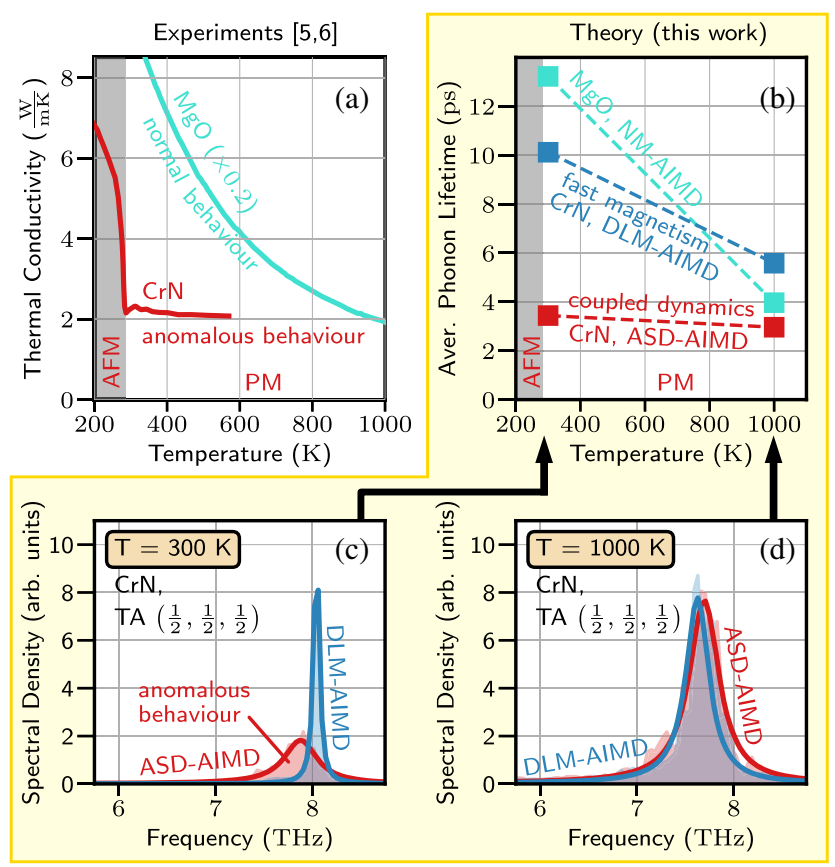

FIG. 1. (a) Thermal conductivities of $\mathrm{CrN}$ [5] and $\mathrm{MgO}[6]$ compared to (b) averaged phonon lifetimes obtained by DLMAIMD, ASD-AIMD and nonmagnetic AIMD. (c) $\mathrm{CrN}$ spectral density of the transversal acoustic phonon at the $L$ point at $T=300 \mathrm{~K}$ and (d) $T=1000 \mathrm{~K}$. Shaded areas indicate the corresponding nonfitted spectral densities. 
magnetization and long range order between the magnetic moments. Nevertheless, local moments with a degree of short-range correlations, both in time and space, may still exist and influence physical properties [9-11]. Furthermore, the lattice degrees of freedom (d.o.f.) experience thermal excitations, often well beyond the harmonic regime [12-14]. Recently, it has been demonstrated by first-principles calculations that the lattice and magnetic excitations are interconnected in the paramagnetic phases of Fe: The interatomic forces that dictate the vibrations and the phonon spectra are distinctly different in the disordered magnetic state as compared to the ferromagnetic ground state $[15,16]$. The local magnetic moments and electronic structure are in turn clearly influenced by the presence of atomic disorder caused by the lattice vibrations [17].

Previous approaches $[15,16,18]$, however, lack an incorporation of the full mutual coupling between both d.o.f. For instance, in the previously proposed spin-space averaging approach $[15,16]$, the atomic fluctuations do not directly impact the magnetic system. In the disordered local moment ab initio molecular dynamics (DLM-AIMD) [18] approach, magnetic short and long range ordering effects are ignored. Properties such as the experimentally observed anomalous thermal conductivity in $\mathrm{CrN}$ cannot be interpreted by these methods.

An alternative would be spin-lattice dynamics (SLD) [19-23], but efficient implementations require adequate interatomic potentials for the magnetic and atomic d.o.f. Constructing these is particularly challenging for magnetic materials at high temperatures. A key prerequisite for such a method development is thus an unbiased and fully firstprinciples based approach.

In this Letter, we suggest an approach to close the previous simulation gap for magnetic materials by a firstprinciples based methodology. Our approach combines atomistic spin dynamics [24,25] with ab initio molecular dynamics (ASD-AIMD). Ab initio molecular dynamics calculations have been successfully applied for a quantitative description of nonmagnetic materials at high temperatures [14]. Likewise, spin dynamics calculations are used to study properties of magnetic materials below and above their magnetic transition temperature [26,27]. Our ASD-AIMD approach now paves the way for the investigation of magnetic materials with AIMD-level accuracy and the explicit inclusion of coupled dynamical excitations at high temperatures. Based on this approach, we derive magnetic and phonon lifetimes in $B 1 \mathrm{CrN}$ above the magnetic ordering temperature of $280 \mathrm{~K}$.

Our ASD-AIMD method is sketched in Fig. 2. It is based on an alternating scheme of ASD and AIMD steps. To start with suitable initial atomic positions, adiabatic DLMAIMD simulations are performed at the temperature of choice. The resulting positions $\{\mathbf{R}\}$ of this pre-equilibration are used to obtain the distance dependent exchange constants $J_{i j}$ between magnetic atoms, which are parametrized prior to the simulation. A reasonable initial spin state $\{\mathbf{S}\}$ is then determined by a Monte Carlo (MC) simulation for these atomic positions and corresponding exchange interactions. After this initiation, the main loop of the ASD-AIMD is started. The spin orientations are used for the subsequent AIMD step. There, forces acting on each atom are determined via a spin polarized, noncollinear density functional theory (DFT) calculation with constrained spin directions consistent with the actual orientation of the spins. The obtained forces are used to update the atomic positions by a single-step propagation of $1 \mathrm{fs}$. With the new atomic positions, new distance dependent exchange interactions are obtained and used to propagate the magnetic state for $1 \mathrm{fs}$ in the ASD simulation of the same coupled ASD-AIMD step. The alternating AIMD and ASD steps are repeated until a sufficient simulation time has been obtained to ensure convergence of the investigated physical quantities.

The propagation of the atoms has been realized by a Born-Oppenheimer type AIMD within VASP [28-33]. The forces acting on the atoms are determined in formally $0 \mathrm{~K}$

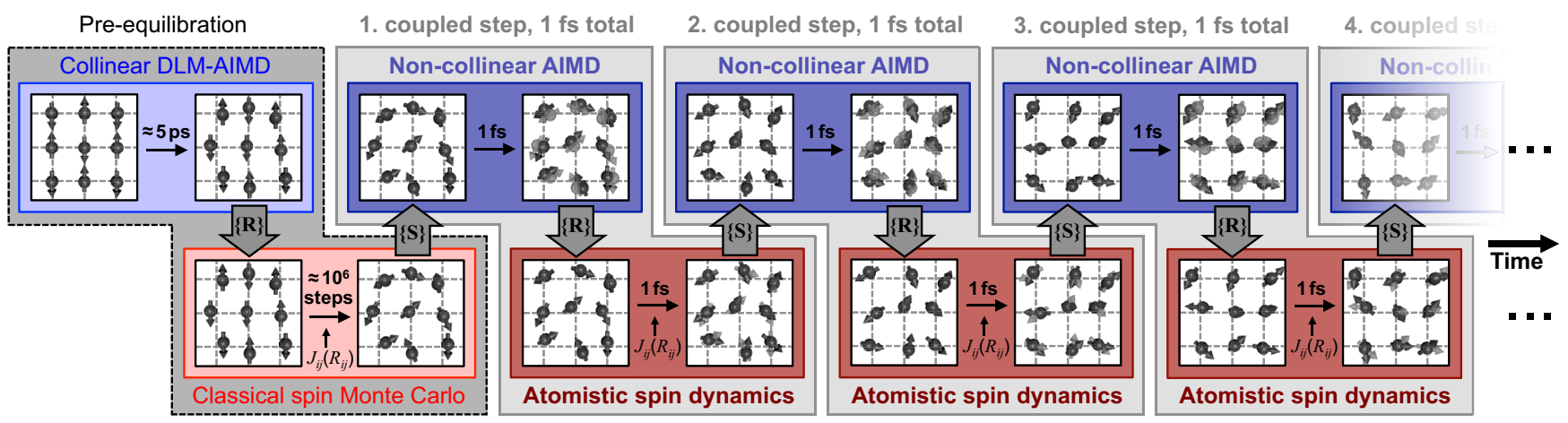

FIG. 2. Representation of the combined ASD-AIMD approach: After a preequilibration, a subsequent scheme of ASD and AIMD runs are performed. At each step, the actual spin state $\{\mathbf{S}\}$ and the actual atomic positions $\{\mathbf{R}\}$ serve as input of the following AIMD and ASD calculation, respectively. Within each coupled step, the atoms and spins propagate for $1 \mathrm{fs}$ in total. 
DFT calculations with a constrained magnetic moment approach by Ma and Dudarev [34] via Lagrange multipliers of magnitude $\lambda=25$. The actual change of the spin state is calculated by the Landau-Lifshitz-Gilbert equation (LLG) as implemented in UPPASD [25,35],

$$
\begin{aligned}
\frac{\partial \hat{\mathbf{S}}_{i}}{\partial t}= & -\frac{\gamma}{1+\alpha^{2}} \hat{\mathbf{S}}_{i} \times\left[\mathbf{H}_{\mathrm{eff}}+\mathbf{f}_{i}\right] \\
& -\gamma \frac{\alpha}{1+\alpha^{2}} \hat{\mathbf{S}}_{i} \times\left\{\hat{\mathbf{S}}_{i} \times\left[\mathbf{H}_{\mathrm{eff}}+\mathbf{f}_{i}\right]\right\} .
\end{aligned}
$$

The quantities $\gamma$ and $\alpha$ denote the electron gyromagnetic ratio and the phenomenological damping factor, respectively. The spin temperature is described by white-noise Langevin dynamics. In these dynamics, the fluctuations are enforced by an auxiliary stochastic magnetic field $\mathbf{f}_{i}$. Both parts of Eq. (1) are based on the effective field $\mathbf{H}_{\text {eff }}=-\left(1 / m_{i}\right)\left[(\partial \mathcal{H}) /\left(\partial \hat{\mathbf{S}}_{i}\right)\right]$, with $m_{i}$ denoting the magnitude of the magnetic moment corresponding to the $i$ th atom. The effective field is determined by the classical Heisenberg exchange interaction between the effective spin of $\mathrm{Cr}$ atoms:

$$
\mathcal{H}=-\sum_{i \neq j} J_{i j}\left(R_{i j}\right) \hat{\mathbf{S}}_{i} \hat{\mathbf{S}}_{j} .
$$

The lattice vibrations enter the spin dynamics through their effect on the exchange interactions $J_{i j}\left(R_{i j}\right)$. The CrN exchange interactions have been determined in [36], and they depend mostly on the $\mathrm{Cr}-\mathrm{Cr}$ atomic pair distances. The range of interactions in paramagnetic $\mathrm{CrN}$ was found to be very short and could be well described with only first and second $\mathrm{Cr}-\mathrm{Cr}$ neighbor terms $[36,37]$. The parametrization of these interactions are determined by magnetic direct cluster averaging using the same electronic structure framework and approximations as used for our AIMD runs [36] (see Supplemental Material [38]). Within our notation, the magnitudes of the effective magnetic moments $m_{i}$ and $m_{j}$ are absorbed into the exchange interactions $J_{i j}\left(R_{i j}\right)$. Performing test calculations, we find spin-orbit coupling effects to be negligibly weak in $\mathrm{CrN}$.

While $\mathrm{N}$ carries a negligible magnetic moment, the magnitude of $\mathrm{Cr}$ moments in $\mathrm{CrN}$ has been observed to be robust around $2.8 \mu_{B}$, with respect to both transversal magnetic disorder [43] and lattice vibrations [44]. We therefore constrain the transverse, orientational, d.o.f. in the LLG equation only. The absolute magnitude of each moment is allowed to adjust freely within each electronic structure step. As discussed in the Supplemental Material [38], the phenomenological damping factor has a small influence on the spin dynamics in equilibrium simulations of the high temperature phase, and the typical value of 0.05 has been chosen [45]. We applied our method to $\mathrm{CrN}$ to investigate the properties within the paramagnetic phase at $T=300 \mathrm{~K}$ and $T=1000 \mathrm{~K}$. Detailed information of DFT related parameters (e.g., $k$ points, energy cutoff, cell sizes) are given in the Supplemental Material [38].

To analyze the importance of a spin-phonon coupling, we performed DLM-AIMD [18] calculations employing the same 1 fs AIMD time step but with fully random, disordered noncollinear magnetic states, changing likewise, each $1 \mathrm{fs}$. In this way, we approximate the adiabatic limit where individual spin states live too short to be "seen" by the atomic vibrations. This can be regarded as a generalization of previous DLM-AIMD works on $\mathrm{CrN}[18,46]$. It considers noncollinear disorder in the limit of an infinitely fast change of magnetic states. All other details of the DLM-AIMD simulations are identical to the ASDAIMD runs.

The most compelling finding is observed for the phonon lifetimes of $\mathrm{CrN}$ in the paramagnetic range (see Supplemental Material [38] for a description on obtaining phonon lifetimes from MD). In Figs. 1(c) and 1(d), we show the power spectral density of the transversal acoustic phonon at the $L$ point as a representative example. There, we compare the coupled ASD-AIMD simulation to the adiabaticlike DLM-AIMD, both at (a) $300 \mathrm{~K}$ and (b) $1000 \mathrm{~K}$. We observe small effects on the phonon frequencies (corresponding to the peak positions). However, the full widths at half maximum $\left(\mathrm{FWHM}=2 \Gamma_{\mathrm{ph}}\right)$, which are inversely proportional to the phonon lifetimes $\left(\tau=\left(1 / 2 \Gamma_{\mathrm{ph}}\right)\right)$, are distinctly different with significantly broader distributions for the coupled ASDAIMD simulation. This effect is particularly apparent at $300 \mathrm{~K}$ and largely vanishes at $1000 \mathrm{~K}$. This impressively demonstrates that at $300 \mathrm{~K}$, above the magnetic transition temperature, the vibrational state is heavily disturbed by the dynamical coupling to the magnetic state without long range order. In panel (b) of Fig. 1, we show the average of all obtained phonon lifetimes for $\mathrm{CrN}$ simulated with the two methods, as well as for the isostructural nonmagnetic $\mathrm{MgO}$. The absolute value at $300 \mathrm{~K}$ and the temperature trend of the averaged phonon lifetimes are distinctly different between the adiabaticlike DLM-AIMD and the dynamically coupled ASD-AIMD simulations. The adiabaticlike simulation for $\mathrm{CrN}$ shows a striking resemblance with the results for nonmagnetic simulations for $\mathrm{MgO}$. The similar temperature trend indicates that disordered magnetism, treated as adiabatically fast on the time scale of the phonons, has little influence on the phonon lifetime and also on their temperature dependence. In contrast, the results for the ASD-AIMD simulation show that when the spin dynamics are explicitly treated and coupled with lattice dynamics, it can indeed drastically decrease phonon lifetimes. Our results therefore show that the coupling strength is large at $300 \mathrm{~K}$ and that it decreases with temperature. Decomposing the phonons into acoustic and optical branches (cf. Supplemental Material [38]), we find that the difference between the DLM and ASD treatments originates from effects on the acoustic branches, while the lifetimes of the optical branches are very similar. This may be expected as the optical modes are governed by 
the interatomic forces of neighboring $\mathrm{Cr}-\mathrm{N}$ pairs, where the $\mathrm{N}$ atoms are almost absent of spin polarization and, in particular, fully absent of spin dynamics.

Since the phonon lifetimes are directly related to the lattice thermal conductivity, these findings provide an intuitive explanation of the constant $[4,5]$ or even increasing [3] thermal conductivity with temperature in the paramagnetic state of $\mathrm{CrN}$, as shown by the measured data from [5] in Fig. 1(a). Normally phonon lifetimes reduce with increasing temperature due to increased phonon-phonon scattering. As discussed above, the dynamic coupling between spin dynamics and lattice vibrations has the opposite temperature dependence-it becomes weaker with increasing temperature. The reduction of the acoustic phonon lifetimes is strong close to the Néel temperature compared to the case of phonon-phonon interaction only. In contrast, at high temperatures, the coupled dynamics have little impact on the phonon lifetimes. As a consequence of this compensating behavior, the phonon lifetime becomes almost temperature independent.

The success of the ASD-AIMD approach to reveal the strong impact of dynamical coupling on the lattice thermal conductivity in $\mathrm{CrN}$ suggests that it is an ideal tool to also address phenomena such as magnetostriction, ultrafast relaxations, or the magnetocaloric effect.

To obtain a detailed understanding of the nature of the coupling effect and its change with temperature, we study (i) the correlation between the momentaneous interatomic distance and spin state (Fig. 3) and (ii) the influence of the lattice vibrations on the magnetic short range order autocorrelations (Fig. 4). The spin dot product $\hat{\mathbf{S}}_{i} \cdot \hat{\mathbf{S}}_{j}$, as a function of the $\mathrm{Cr}$-Cr nearest-neighbor pair distance $R_{i j}$, is shown in Fig. 3 as a histogram for every AIMD snapshot. The histogram represents the probability in finding a pair with a specific distance and spin orientation. Including

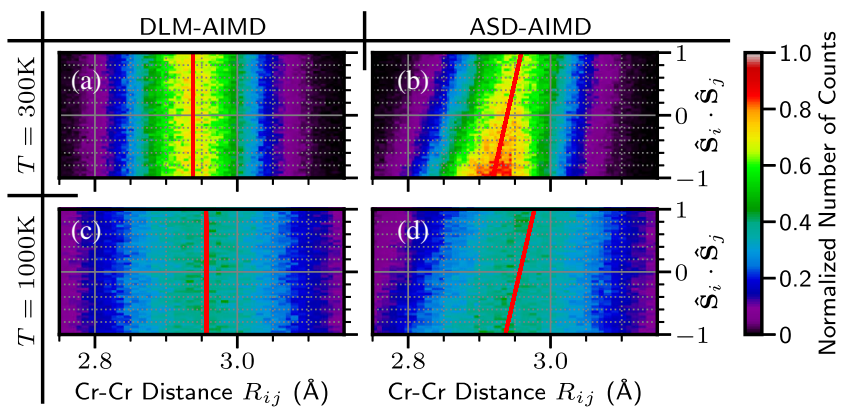

FIG. 3. Two dimensional probability diagrams of $\mathrm{Cr}-\mathrm{Cr}$ nearest neighbor pairs with respect to their spin orientation. The spin orientation is given by the dot products of the two spin vectors in a pair [1 (-1) corresponds to the ferro- (antiferro-) magnetic coupling]. The diagrams are plotted for different temperatures and methods: (a) DLM-AIMD at $T=300 \mathrm{~K}$, (b) ASD-AIMD at $T=300 \mathrm{~K}$, (c) DLM-AIMD at $T=1000 \mathrm{~K}$, and (d) ASDAIMD at $T=1000 \mathrm{~K}$. The red lines show the linear regression between distances and spin orientations. dynamic coupling [Figs. 3(b) and 3(d)], a distinct correlation between distances and spin orientations is observed. Specifically, the AFM oriented pairs have a tendency for shorter distances, while FM oriented pairs have a tendency for longer distances. The higher probability at antiferromagnetic orientations (indicated by the red color) implies that the system shows substantial antiferromagnetic short range order (SRO) at $300 \mathrm{~K}$. At a higher temperature of $1000 \mathrm{~K}$ [Fig. 3(d)], local spin order largely disappears, and the correlation between pair separation and spin orientation is less pronounced relative to the larger total spread in pair distances. As expected, when not taking the dynamic coupling into account [Figs. 3(a) and 3(c)], no correlation is observed. In order to verify that the spin-lattice correlation is indeed due to the dynamical coupling and not due to spatial magnetic SRO, we replace the spin-dynamic step in our approach by Monte Carlo (MC) calculations independent from each other but including magnetic SRO. The comparison clearly shows, Figs. 5 and 6 in the Supplemental Material [38], a magnetic SRO at $300 \mathrm{~K}$ without impact on interatomic distances or suppression of phonon lifetimes, proving the dynamical origin of the latter effects.

Having studied the impact of the spin dynamics on the dynamics of the nuclei, we now study the opposite effect; i.e., the impact of the atomic vibrations on spin dynamics. For this reason we consider three cases. In the first case, we keep the atoms fixed on their equilibrium positions; i.e., by construction the atomic dynamics is suppressed. Second, we switch on atomic dynamics, but we do not couple it to the spin dynamics. Nevertheless, the spin dynamics are influenced by the distances between magnetic atoms. Third, we consider the fully coupled case. As shown in Fig. 4, including atomic dynamics always reduces the spin correlation time. The magnitude of this decrease depends significantly on the temperature: While the shortening of spin correlation times is modest at low temperatures, it becomes large at high temperatures. What is interesting is the qualitatively different impact of the dynamical coupling. While at high temperatures there is no visible

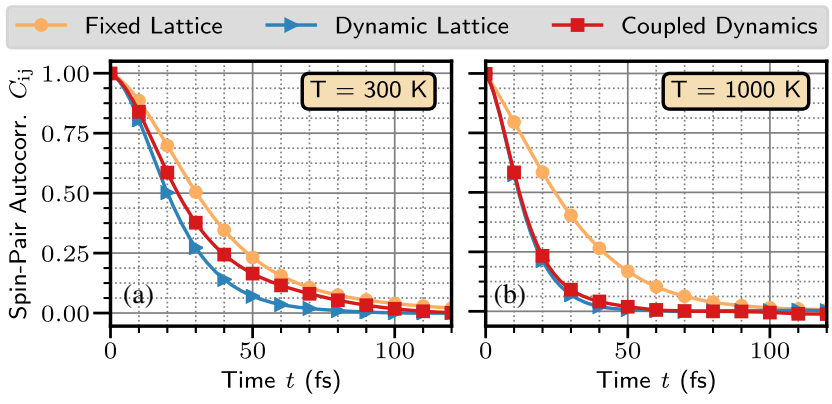

FIG. 4. Spin pair autocorrelation functions of $\mathrm{CrN}$ at $T=$ $300 \mathrm{~K}$ (a) and $T=1000 \mathrm{~K}$ (b) obtained from ASD (fixed lattice), ASD on a pre-calculated DLM-AIMD vibrating lattice (dynamic lattice) and ASD-AIMD (coupled dynamics). 
difference between coupled and noncoupled atom dynamics, at low temperatures there are clear differences. Including dynamical coupling between the spin and lattice increases the spin correlation times; i.e., spin scattering at the vibrating atoms becomes less efficient.

The weaker spin-lattice coupling at $1000 \mathrm{~K}$ than at $300 \mathrm{~K}$ can be understood by two effects. First, the even shorter lifetimes of the magnetic state at $1000 \mathrm{~K}$, seen in Fig. 4, brings the system towards an adiabatic decoupling. Second, the higher amplitude of atomic displacements, seen in the distribution width in Fig. 3, corresponds to an increased vibrational energy scale not matched by an increase in the energy scale of the magnetic fluctuations that, already at $300 \mathrm{~K}$, is in the disordered paramagnetic regime.

In conclusion, we have proposed a method that allows us to simulate the high temperature paramagnetic phase of magnetic materials by combining ab initio parametrized atomistic spin dynamics with ab initio molecular dynamics. The approach enables studies of the dynamical coupling of spin and lattice excitations and its impact on the properties of materials. For the model system studied, $\mathrm{CrN}$, we find that the dynamics of the spin system severely impacts atomic lattice vibrations and the quantities related to it, such as phonon lifetimes. The dependence is mutual, and the magnetic state and its dynamic are influenced by the lattice vibrations. At temperatures slightly above the transition temperature, the dynamical coupling, which is accessible by our approach, is found to significantly reduce the phonon lifetimes of the acoustic modes. In contrast, at high temperatures $(1000 \mathrm{~K})$ well above the Néel temperature, the dynamical coupling and its impact on phonon lifetimes are largely reduced. Thus the strong spin-lattice coupling identified in this Letter and the fact that it is found to decrease with increasing temperature, is consistent with experimental observations. We expect that similar mechanisms apply not only for $\mathrm{CrN}$ but for a wide range of magnetic semiconductors and oxides, e.g., $\mathrm{YMnO}_{3}$, $\mathrm{LuMnO}_{3}$, and $\mathrm{ScMnO}_{3}$, since related experimental findings have been observed. Furthermore, the application of the ASD-AIMD method beyond magnetic semiconductors will allow us to explore the impact of dynamical spin-lattice coupling on the properties of magnetic materials at high temperature.

A. B. acknowledges the CEA-Enhanced Eurotalents program, cofunded by FP7 Marie Skłodowska-Curie COFUND Programme (Grant Agreement No. 600382), T. H. acknowledges the priority programme SPP1599 "Ferroic cooling" (Grant No. HI1300/6-2), F. K. acknowledges funding by the Netherlands Organisation for Scientific Research (NWO) under the VIDI research programme, with Project No. 15707. B. G. acknowledges funding by the European Research Council (ERC) under the EU's Horizon 2020 Research and Innovation Programme (Grant No. 639211). B. A. acknowledges financial support by the Swedish Research Council (VR) through the International Career
Grant No. 330-2014-6336 and by Marie Skłodowska Curie Actions, Cofund, Project INCA 600398, the Swedish Government Strategic Research Area in Materials Science on Functional Materials at Linköping University (Faculty Grant SFOMatLiU No. 2009 00971), as well as support from the Swedish Foundation for Strategic Research through the Future Research Leaders 6 program. Per Eklund is acknowledged for useful discussions.

*irina.stockem@liu.se

[1] J. Fransson, D. Thonig, P. F. Bessarab, S. Bhattacharjee, J. Hellsvik, and L. Nordström, Phys. Rev. Mater. 1, 074404 (2017).

[2] P. A. Sharma, J. S. Ahn, N. Hur, S. Park, S. B. Kim, S. Lee, J.-G. Park, S. Guha, and S.-W. Cheong, Phys. Rev. Lett. 93, 177202 (2004).

[3] C. X. Quintela, F. Rivadulla, and J. Rivas, Appl. Phys. Lett. 94, 152103 (2009).

[4] P. Tomeš, D. Logvinovich, J. Hejtmánek, M. H. Aguirre, and A. Weidenkaff, Acta Mater. 59, 1134 (2011).

[5] O. Jankovský, D. Sedmidubský, Š. Huber, P. Šimek, and Z. Sofer, J. Eur. Ceram. Soc. 34, 4131 (2014).

[6] R. Powell, C. Ho, and P. Liley, Thermal Conductivity of Selected Materials, NSRDS-NBS (U.S. Department of Commerce, National Bureau of Standards, Washington, D.C., 1968), Part 2.

[7] A. Togo, L. Chaput, and I. Tanaka, Phys. Rev. B 91, 094306 (2015).

[8] G. Fugallo and L. Colombo, Phys. Scr. 93, 043002 (2018).

[9] F. Rivadulla, M. Banobre-Lopez, C. X. Quintela, A. Pineiro, V. Pardo, D. Baldomir, M. A. Lopez-Quintela, J. Rivas, C. A. Ramos, H. Salva, J.-S. Zhou, and J. B. Goodenough, Nat. Mater. 8, 947 (2009).

[10] B. Alling, T. Marten, and I. A. Abrikosov, Nat. Mater. 9, 283 (2010).

[11] S. Wang, X. Yu, J. Zhang, M. Chen, J. Zhu, L. Wang, D. He, Z. Lin, R. Zhang, K. Leinenweber, and Y. Zhao, Phys. Rev. B 86, 064111 (2012).

[12] Y. Ikeda, F. Körmann, B. Dutta, A. Carreras, A. Seko, J. Neugebauer, and I. Tanaka, npj Comput. Mater. 4, 7 (2018).

[13] R. Pradip, P. Piekarz, A. Bosak, D. G. Merkel, O. Waller, A. Seiler, A. I. Chumakov, R. Rüffer, A. M. Oleś, K. Parlinski, M. Krisch, T. Baumbach, and S. Stankov, Phys. Rev. Lett. 116, 185501 (2016).

[14] A. Glensk, B. Grabowski, T. Hickel, and J. Neugebauer, Phys. Rev. Lett. 114, 195901 (2015).

[15] F. Körmann, A. Dick, B. Grabowski, T. Hickel, and J. Neugebauer, Phys. Rev. B 85, 125104 (2012).

[16] F. Körmann, B. Grabowski, B. Dutta, T. Hickel, L. Mauger, B. Fultz, and J. Neugebauer, Phys. Rev. Lett. 113, 165503 (2014).

[17] B. Alling, F. Körmann, B. Grabowski, A. Glensk, I. A. Abrikosov, and J. Neugebauer, Phys. Rev. B 93, 224411 (2016).

[18] P. Steneteg, B. Alling, and I. A. Abrikosov, Phys. Rev. B 85, 144404 (2012).

[19] P.-W. Ma, C. H. Woo, and S. L. Dudarev, Phys. Rev. B 78, 024434 (2008). 
[20] P.-W. Ma, S. Dudarev, and C. Woo, Comput. Phys. Commun. 207, 350 (2016).

[21] P.-W. Ma, S. L. Dudarev, and J. S. Wróbel, Phys. Rev. B 96, 094418 (2017).

[22] D. Perera, M. Eisenbach, D. M. Nicholson, G. M. Stocks, and D. P. Landau, Phys. Rev. B 93, 060402 (2016).

[23] D. Perera, D. M. Nicholson, M. Eisenbach, G. M. Stocks, and D. P. Landau, Phys. Rev. B 95, 014431 (2017).

[24] V. P. Antropov, M. I. Katsnelson, B. N. Harmon, M. van Schilfgaarde, and D. Kusnezov, Phys. Rev. B 54, 1019 (1996).

[25] B. Skubic, J. Hellsvik, L. Nordström, and O. Eriksson, J. Phys. Condens. Matter 20, 315203 (2008).

[26] X. Tao, D. P. Landau, T. C. Schulthess, and G. M. Stocks, Phys. Rev. Lett. 95, 087207 (2005).

[27] J. Hellsvik, B. Skubic, L. Nordström, B. Sanyal, O. Eriksson, P. Nordblad, and P. Svedlindh, Phys. Rev. B 78, 144419 (2008).

[28] G. Kresse and J. Hafner, Phys. Rev. B 47, 558 (1993).

[29] G. Kresse and J. Furthmüller, Comput. Mater. Sci. 6, 15 (1996).

[30] G. Kresse and J. Furthmüller, Phys. Rev. B 54, 11169 (1996).

[31] G. Kresse and D. Joubert, Phys. Rev. B 59, 1758 (1999).

[32] P. E. Blöchl, Phys. Rev. B 50, 17953 (1994).

[33] D. Hobbs, G. Kresse, and J. Hafner, Phys. Rev. B 62, 11556 (2000).

[34] P.-W. Ma and S. L. Dudarev, Phys. Rev. B 91, 054420 (2015).
[35] O. Eriksson, A. Bergman, L. Bergqvist, J. Hellsvik, and O. U. Press, Atomistic Spin Dynamics: Foundations and Applications (Oxford University Press, Oxford, 2017).

[36] A. Lindmaa, R. Lizárraga, E. Holmström, I. A. Abrikosov, and B. Alling, Phys. Rev. B 88, 054414 (2013).

[37] A. Lindmaa, Master's thesis, Linköping University, 2012.

[38] See Supplemental Material at http://link.aps.org/ supplemental/10.1103/PhysRevLett.121.125902 for the computational parameters of the ASD and AIMD calculations, additional information on the autocorrelation functions of the magnetic moments, and the equations for thephonon analysis, and supporting additional results of Monte Carlo AIMD, as vwell as decomposition of phonon lifetimes in acoustic and optical modes, which includes Refs. [39-42].

[39] D. M. Ceperley and B. J. Alder, Phys. Rev. Lett. 45, 566 (1980).

[40] S. L. Dudarev, G. A. Botton, S. Y. Savrasov, C. J. Humphreys, and A. P. Sutton, Phys. Rev. B 57, 1505 (1998).

[41] B. Alling, T. Marten, and I. A. Abrikosov, Phys. Rev. B 82, 184430 (2010).

[42] J. P. Perdew, K. Burke, and M. Ernzerhof, Phys. Rev. Lett. 77, 3865 (1996).

[43] B. Alling, Phys. Rev. B 82, 054408 (2010).

[44] N. Shulumba, B. Alling, O. Hellman, E. Mozafari, P. Steneteg, M. Odén, and I. A. Abrikosov, Phys. Rev. B 89, 174108 (2014).

[45] S. Mizukami, J. Magn. Soc. Jpn. 39, 1 (2015).

[46] E. Mozafari, N. Shulumba, P. Steneteg, B. Alling, and I. A. Abrikosov, Phys. Rev. B 94, 054111 (2016). 